\title{
A shifting approach to management of the thoracic aorta in bicuspid aortic valve
}

\author{
Alexander R. Opotowsky, MD, MPH, ${ }^{\mathrm{a}, \mathrm{b}}$ Todd Perlstein, MD, ${ }^{\mathrm{b}}$ Michael J. Landzberg, MD, ${ }^{\mathrm{a}, \mathrm{b}}$ \\ Steven D. Colan, MD, ${ }^{\mathrm{a}}$ Patrick T. O'Gara, MD, ${ }^{\mathrm{b}}$ Simon C. Body, MBChB, MPH, ${ }^{\mathrm{c}}$ Liam F. Ryan, MD, ${ }^{\mathrm{d}}$ \\ Sary Aranki, MD, ${ }^{\mathrm{e}}$ and Michael N. Singh, MD ${ }^{\mathrm{a}, \mathrm{b}}$
}

\begin{abstract}
Objectives: The scientific understanding of aortic dilation associated with bicuspid aortic valve (BAV) has evolved during the past 2 decades, along with improvements in diagnostic technology and surgical management. We aimed to evaluate secular trends and predictors of thoracic aortic surgery among patients with BAV in the United States.
\end{abstract}

\begin{abstract}
Methods: We used the 1998-2009 Nationwide Inpatient Sample, an administrative dataset representative of US hospital admissions, to identify hospitalizations for adults aged 18 years or more with BAV and aortic valve or thoracic aortic surgery. Covariates included age, gender, year, aortic dissection, endocarditis, thoracic aortic aneurysm, number of comorbidities, hospital teaching status and region, primary insurance, and concomitant coronary artery bypass surgery.

Results: Between 1998 and 2009, 48,736 \pm 3555 patients with BAV underwent aortic valve repair or replacement and $1679 \pm 120$ patients with BAV underwent isolated thoracic aortic surgery. The overall number of surgeries increased more than 3 -fold, from $4556 \pm 571$ in $1998 / 1999$ to $14,960 \pm 2107$ in 2008/2009 $(P<.0001)$. The proportion of aortic valve repair or replacement including concomitant thoracic aortic surgery increased from $12.8 \% \pm 1.4 \%$ in $1998 / 1999$ to $28.5 \% \pm 1.6 \%$ in $2008 / 2009$, which mirrored an increasing proportion of patients with a diagnosis of thoracic aortic aneurysm. Mortality was equivalent for patients undergoing aortic valve repair or replacement with thoracic aortic surgery and those undergoing isolated aortic valve repair or replacement $(1.8 \% \pm 0.3 \%$ vs $1.5 \% \pm 0.2 \%$; multivariable odds ratio, $1.02 ; 95 \%$ confidence interval, $0.67-1.57)$, with decreasing mortality over the study period (from $2.5 \% \pm 0.6 \%$ in $1998 / 1999$ to $1.5 \% \pm 0.2 \%$ in 2008 / 2009; multivariable odds ratio per 2-year increment, $0.89 ; 95 \%$ confidence interval, $0.81-0.99 ; P=.03$ ). Total charges for BAV surgical hospitalizations increased more than 7.5-fold from approximately $\$ 156$ million in 1998 to $\$ 1.2$ billion in 2009 (inflation-adjusted 2009 dollars).
\end{abstract}

Conclusions: There was a marked increase in the use of thoracic aortic surgery among patients with BAV. (J Thorac Cardiovasc Surg 2013;146:339-46)

Bicuspid aortic valve (BAV) is the most common congenital valvular heart defect, being present in approximately $1 \%$ of the general population. ${ }^{1-3} \mathrm{BAV}$ is associated with an increased risk of aortic valve stenosis and regurgitation, as

\footnotetext{
From the Department of Cardiology, ${ }^{\text {a }}$ Boston Children's Hospital, Boston, Mass; Division of Cardiovascular Medicine, ${ }^{\mathrm{b}}$ Department of Medicine, Brigham \& Women's Hospital, Boston, Mass; Department of Anesthesiology, ${ }^{\mathrm{c}}$ Perioperative \& Pain Medicine, Brigham \& Women's Hospital, Boston, Mass; Sanger Heart and Vascular Institute, ${ }^{\mathrm{d}}$ Carolinas Medical Center, Charlotte, NC; and Division of Cardiovascular Surgery, ${ }^{\mathrm{e}}$ Department of Surgery, Brigham \& Women's Hospital, Boston, Mass.

Funding: Supported by National Institutes of Health Grants 5 T32 HL07604-25 and UL1 RR 025758, and the Dunlevie Fund.

Disclosures: Authors have nothing to disclose with regard to commercial support.

Received for publication July 8, 2012; revisions received Sept 21, 2012; accepted for publication Oct 16, 2012; available ahead of print Nov 12, 2012.

Address for reprints: Alexander R. Opotowsky, MD, MPH, Boston Adult Congenital Heart Service, Department of Cardiology, Brigham and Women's Hospital, Boston Children's Hospital, 300 Longwood Ave, Bader 209, Boston, MA 02115 (E-mail: alexander.opotowsky@childrens.harvard.edu).

$0022-5223 / \$ 36.00$

Copyright (c) 2013 by The American Association for Thoracic Surgery

http://dx.doi.org/10.1016/j.jtcvs.2012.10.028
}

well as thoracic aortic dilation and dissection. Aortic dilation was long considered to be due to valve disease itself, but increasing evidence points to an independent aortopathy associated with BAV. ${ }^{3-6}$ Although the relative risk of aortic dissection is lower than in Marfan syndrome, there are likely as many, if not more, dissections in patients with BAV given the significantly greater prevalence of this disease. ${ }^{7}$ Therefore, BAV-associated aortic disease has important public health implications.

Surgical management of aortic dilation and aneurysm presents a difficult clinical problem given the unpredictable lifetime risk of morbidity and mortality ${ }^{7-9}$ related to BAV aortopathy and major surgical intervention required to address these risks. This is not a new clinical issue; the complexities of aortic aneurysmal disease have long been appreciated, ${ }^{10,11}$ but our understanding and ability to diagnose and intervene have evolved considerably. Recommendations on when to intervene surgically for thoracic aortic dilation, in general and for patients with $\mathrm{BAV}$, have been progressively expanded over the past 15 


\author{
Abbreviations and Acronyms \\ $\mathrm{ACC}=$ American College of Cardiology \\ $\mathrm{AHA}=$ American Heart Association \\ $\mathrm{AVR}=$ aortic valve repair or replacement \\ $\mathrm{BAV}=$ bicuspid aortic valve \\ $\mathrm{CI}=$ confidence interval \\ OR $=$ odds ratio \\ $\mathrm{TAA}=$ thoracic aortic aneurysm \\ TAS $=$ thoracic aortic surgery
}

years. Indeed, BAV is now widely considered to be an independent risk factor for an acute aortic event, leading to a lower threshold for aortic surgery in patients with BAV compared with "degenerative" thoracic aortic aneurysms (TAAs). ${ }^{12}$ Bicuspid aortopathy is increasingly thought of as a genetic disease affecting aortic structure and metabolism, with some arguing that aortic dimensions indicating surgical intervention should be similar to those used for other such genetic diagnoses, such as Marfan syndrome. ${ }^{13-15}$ Absolute aortic diameter remains the most used clinical parameter to guide intervention, although indexed and nonsize predictors also have been proposed. ${ }^{16-18}$ Guideline recommendations for surgical intervention based on a threshold of ascending aortic diameter have decreased from more than $5 \mathrm{~cm}^{19-22}$ to more than $4.5 \mathrm{~cm}$ for patients with BAV undergoing concomitant aortic valve repair or replacement (AVR), with others proposing even lower thresholds for intervention. ${ }^{16,23,24}$ However, these recommendations remain controversial. ${ }^{9,25,26}$

This study aims to elucidate practice patterns in the surgical management of the thoracic aorta in patients with BAV by examination of a representative dataset of US hospitalizations over a recent 12-year period.

\section{MATERIALS AND METHODS}

We used the 1998-2009 Nationwide Inpatient Sample (Healthcare Cost and Utilization Project, Agency for Healthcare Research and Quality), the largest all-payer nationally representative hospital discharge database in the United States, to investigate the epidemiology of AVR and thoracic aortic surgery (TAS) among adults (aged $\geq 18$ years) with BAV. This database has been used to study other aspects of hospitalizations for congenital heart disease and noncongenital cardiac surgery. ${ }^{27-30}$ Because this study used publicly available anonymous data, the institutional review board of Brigham and Women's Hospital granted exemption from review.

The primary aims of the study were to describe temporal trends in the incidence of TAS and AVR (surgical repair/replacement or endovascular repair) among patients with BAV. We collated patients hospitalized with an International Classification of Diseases 9th Revision code of BAV (746.4) undergoing concomitant AVR (35.10 or 35.11); AVR (mechanical $=35.22$ or tissue $=35.21$ ) or resection of the thoracic vessel with replacement (38.45); resection of vessel with anastomosis, aorta (38.34); or endovascular implantation of the graft in the thoracic aorta (39.73).

Covariates included age, gender, number of comorbidities as described by Elixhauser and colleagues, ${ }^{31}$ aortic dissection, subacute bacterial endocarditis, diagnosis of TAA, number of comorbidities, year and type of surgery, and concomitant coronary artery bypass surgery or mitral valve repair. Inpatient hospital characteristics ascertained were hospital teaching status, hospital region (Northeast, Midwest, South, and West), and primary insurance for the admission. Hospitalizations were grouped into 2-year increments (1998/1999, 2000/2001, 2002/2003, 2004/2005, 2006/2007, and 2008/2009)

Continuous and categorical variables are presented as mean \pm standard error of the mean and percent \pm standard error percent, respectively. Standard errors of the estimates are presented to show the variance of the estimate when extrapolated from the representative Nationwide Inpatient Sample to the whole US population. Linear regression and logistic regression were used to model continuous dependent variables (eg, total charges, length of stay) and the odds for dichotomous outcomes (eg, TAS, death), respectively. Statistical analyses were performed using SAS for Windows 9.3 (SAS Institute Inc, Cary, NC). All analyses used provided sample weights and accounted for complex sample design and hospital clustering. ${ }^{32,33}$

\section{RESULTS}

Hospitalizations of 50,415 \pm 3671 patients with BAV who underwent AVR or TAS were examined. Demographic and clinical characteristics are detailed in Table 1.

\section{Aortic Valve and Thoracic Aortic Surgery}

Between 1998 and 2009, 48,736 \pm 3555 patients with BAV underwent AVR $(50.3 \% \pm 2.2 \%$ with a mechanical prosthesis; $47.6 \% \pm 1.9 \%$ with a tissue prosthesis), whereas only $2.4 \% \pm 0.5 \%$ underwent valve repair. Of patients undergoing AVR, $22.6 \% \pm 1.0 \%$ underwent concomitant TAS. During the same period, $1679 \pm 162$ patients underwent isolated TAS. An endovascular approach, possibly suggestive of descending thoracic aortic intervention, was applied in $0.4 \% \pm 0.1 \%$ of cases.

During the study period, the overall number of surgeries per 2-year epoch increased 3.3-fold, with the number of isolated AVRs increasing 2.7-fold, the number of AVRs with concomitant TAS increasing 7.3-fold, and the number of isolated TAS increasing 4.5-fold (Figure 1 and Table 2). The proportion of AVRs that included a concomitant thoracic aortic intervention increased from $12.8 \% \pm 1.4 \%$ to $28.5 \% \pm 1.6 \%$ over the study period (Figure 2). Among those who underwent AVR, mechanical valves were implanted in $69.2 \% \pm 3.2 \%$ of patients in $1998 / 1999$, but that number had decreased to just $37.8 \% \pm 3.7 \%$ of patients in 2008/2009 (Table 2). The same trend also was seen in the subset of patients who underwent AVR in conjunction with TAS $(76.1 \% \pm 4.7 \%$ mechanical in $1998 /$ 1999 to $41.0 \% \pm 4.5 \%$ in $2008 / 2009, P<.0001)$. The frequency of mitral valve repair also increased over the study period, but the proportion of patients undergoing mitral repair was lower (Table 2). In contrast, the proportion of patients with BAV who underwent concomitant coronary artery bypass grafting remained unchanged over the study period. 
TABLE 1. Descriptive statistics for patients with bicuspid aortic valve who were hospitalized in the United States between 1998 and 2009 for aortic valve or thoracic aortic surgery

\begin{tabular}{|c|c|c|c|c|c|c|c|c|c|}
\hline & \multicolumn{2}{|c|}{ All } & \multicolumn{2}{|c|}{ AVR only } & \multicolumn{2}{|c|}{ AVR with TAS } & \multicolumn{2}{|c|}{ Isolated TAS } & \multirow[b]{2}{*}{$P$ value } \\
\hline & $\%$ & SE & $\%$ & SE & $\%$ & SE & $\%$ & SE & \\
\hline No. (n), weighted & 50,395 & 3670 & 37,696 & 2564 & 11,020 & 1122 & 1679 & 162 & \\
\hline Age, y (mean, SEM) & 54.1 & 0.3 & 54.7 & 0.3 & 53.1 & 0.4 & 47.1 & 0.9 & $<.0001$ \\
\hline \multicolumn{10}{|l|}{ Age group (y) } \\
\hline $18-30$ & 6.1 & 0.4 & 6.3 & 0.4 & 4.3 & 0.4 & 12.9 & 2.2 & $<.0001$ \\
\hline$>30-40$ & 10.6 & 0.4 & 10.1 & 0.4 & 11.6 & 0.7 & 16.2 & 2.1 & \\
\hline$>40-50$ & 18.9 & 0.5 & 17.5 & 0.6 & 22.5 & 1 & 26.7 & 2.2 & \\
\hline$>50-65$ & 39.3 & 0.5 & 39.1 & 0.6 & 40.9 & 1 & 32.6 & 2.3 & \\
\hline$>65$ & 25.1 & 0.7 & 27 & 0.7 & 20.7 & 1.1 & 11.6 & 1.9 & \\
\hline Gender (female) & 27 & 0.5 & 28.2 & 0.6 & 21.9 & 1 & 31.3 & 2.4 & $<.0001$ \\
\hline Aortic dissection & 2.4 & 0.2 & 0.7 & 0.1 & 6.8 & 0.6 & 11.8 & 1.8 & $<.0001$ \\
\hline SBE & 3.5 & 0.2 & 4.2 & 0.3 & 1.5 & 0.3 & 0.5 & 0.4 & $<.0001$ \\
\hline Coarctation & 1.2 & 0.1 & 0.6 & 0.1 & 0.9 & 0.2 & 14.6 & 2.2 & $<.0001$ \\
\hline CABG & 21.7 & 0.6 & 22.3 & 0.5 & 20.6 & 1.8 & 14.2 & 1.6 & .04 \\
\hline TAA & 26.3 & 1.1 & 7.1 & 0.5 & 84.8 & 1.1 & 72.6 & 2.6 & $<.0001$ \\
\hline Mechanical valve & 50.3 & 2.2 & 50.4 & 2 & 50.2 & 3.2 & & & .94 \\
\hline \multicolumn{10}{|l|}{ Comorbidities } \\
\hline 0 & 39.8 & 1.4 & 38.8 & 1.3 & 42 & 2.3 & 47.7 & 2.7 & $<.0001$ \\
\hline 1 & 31.1 & 0.6 & 30.8 & 0.7 & 32 & 1.1 & 31.4 & 2.6 & \\
\hline 2 & 17.6 & 0.6 & 18 & 0.6 & 16.8 & 1.2 & 13.4 & 2.1 & \\
\hline$\geq 3$ & 11.6 & 0.7 & 12.4 & 0.8 & 9.2 & 1 & 7.5 & 1.5 & \\
\hline Turner syndrome & 0.3 & 0.1 & 0.2 & 0.1 & 0.4 & 0.1 & 2.1 & 0.8 & $<.0001$ \\
\hline Marfan syndrome & 0.3 & 0.1 & 0.1 & 0.04 & 0.7 & 0.2 & 1.5 & 0.7 & $<.0001$ \\
\hline Teaching hospital & 74.4 & 2.4 & 72.7 & 2.4 & 79.1 & 2.9 & 80.7 & 3.7 & .0002 \\
\hline \multicolumn{10}{|l|}{ Hospital region } \\
\hline Northeast & 19 & 2.8 & 18.5 & 2.5 & 21.4 & 4.2 & 15.7 & 3 & $<.0001$ \\
\hline Midwest & 31.1 & 3.6 & 29 & 3.2 & 37.2 & 5.3 & 38 & 4.6 & \\
\hline South & 28.7 & 3.4 & 30.8 & 3.4 & 21.9 & 3.7 & 26 & 4.5 & \\
\hline West & 21.1 & 2.3 & 21.7 & 2.2 & 19.5 & 3.2 & 20.2 & 4.2 & \\
\hline Private insurance & 64.1 & 0.8 & 61.7 & 0.9 & 71 & 1.2 & 73.7 & 2.6 & $<.0001$ \\
\hline Died & 1.6 & 0.1 & 1.5 & 0.2 & 1.8 & 0.3 & 2.9 & 0.9 & .07 \\
\hline
\end{tabular}

There was an increase in the overall number of AVR and TAS performed in patients with BAV in the United States over time. This increase was most marked for patients undergoing AVR and concomitant TAS. AVR, Aortic valve repair or replacement; TAS, thoracic aortic surgery; $S E$, standard error; SEM, standard error of the mean; SBE, subacute bacterial endocarditis; $C A B G$, coronary artery bypass grafting; $T A A$, thoracic aortic aneurysm.

The increase in the proportion of AVR surgeries that included TAS over the study period was independent of age group, gender, and hospital teaching status (Figure 3, $A-C$, respectively) but was mirrored by an increase in the reported diagnosis of TAA, from $15.0 \% \pm 1.8 \%$ of admissions in $1989 / 1990$ to $33.4 \% \pm 1.6 \%$ by $2008 / 2009$; $77.8 \% \pm 1.3 \%$ of those with TAA underwent concomitant TAS.

Among patients undergoing AVR, there was no difference in the frequency of TAS among those without and those with coarctation $(22.6 \% \pm 1.0 \%$ vs $30.4 \% \pm 6.2 \%$, respectively, $P=.15$ ), although patients with coarctation were more likely to undergo isolated TAS $(14.6 \% \pm 2.1 \%$ of isolated aortic surgery was among patients with coarctation, compared with only $1.2 \% \pm 0.1 \%$ of the overall population).

Aortic dissection accounted for a decreasing proportion of surgical BAV admissions, from $4.0 \% \pm 0.7 \%$ to
$2.3 \% \pm 0.3 \%$ over the study period $(P$ for trend $=.003)$. There was no change in the proportion of admissions associated with subacute bacterial endocarditis (overall $3.5 \% \pm 0.2 \% ; P$ for trend $=.16$ ) or coarctation of the aorta (overall $1.2 \% \pm 0.1 \% ; P$ for trend $=.09$ ).

Excluding patients with coarctation, subacute bacterial endocarditis, aortic dissection, Marfan syndrome, and Turner syndrome from the analysis did not affect the observed trends.

\section{Mortality and Resource Use}

There was no difference in unadjusted hospital mortality between those who underwent AVR who had concomitant aortic surgery and those who underwent isolated AVR (odds ratio [OR], 1.22; 95\% confidence interval [CI], 0.83-1.81). Adjustment for clinical covariates similarly failed to find an association (OR, $1.02 ; 95 \% \mathrm{CI}$, 0.67-1.57). There was a trend toward decreasing 


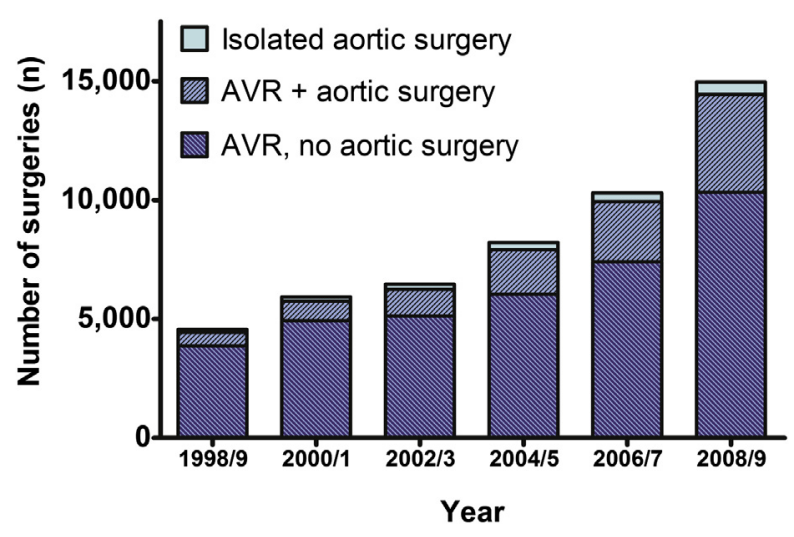

FIGURE 1. The number of surgical procedures performed in patients with BAV in the United States from 1998 to 2009, stratified by procedure type. There was an increase in the overall number of AVR and TAS among patients with BAV in the United States over time. This increase was most marked for patients undergoing AVR and concomitant TAS. AVR, Aortic valve repair or replacement; $T A S$, thoracic aortic surgery; $B A V$, bicuspid aortic valve.

in-hospital mortality over the study period from $2.5 \% \pm 0.6 \%$ in $1998 / 1999$ to $1.5 \% \pm 0.2 \%$ in $2008 /$ 2009 (Table 2 and Figure 4; $P$ for trend .02 ; multivariable OR per 2-year time period, 0.89 ; 95\% CI, 0.81-0.99; $P=.03$ ). Although those undergoing isolated aortic surgery had higher unadjusted mortality than those undergoing AVR alone (OR, 2.04; 95\% CI, 1.11-3.76), this was entirely accounted for by the higher frequency of patients with aortic dissection in the group undergoing isolated aortic surgery (adjusting for dissection OR, 1.07; 95\% CI, 0.53-2.17).

There was no difference in the length of stay for patients undergoing AVR whether they did or did not undergo concomitant TAS (median 5 [6-8] vs 5 [6-9] days, $P=.97$ ) even when adjusted for clinical and hospital covariates and epoch $(P=.39)$. However, unadjusted total hospital charges for those undergoing AVR and TAS were higher than those undergoing isolated AVR ( $\$ 97,370$ vs $\$ 81,589$, respectively, $+\$ 15,780 \pm \$ 3608 ; P<.0001$ ). Adjustment for patient and hospital characteristics, year of surgery, presence of aortic dissection, endocarditis, coarctation, and concomitant coronary artery bypass grafting, as well as medical comorbidities, decreased the cost differential for concomitant TAS to $+\$ 5563 \pm \$ 3281(P=.03)$. Total hospital charges per hospitalization increased approximately $80 \%$, from $\$ 71,861 \pm \$ 2577$ to $\$ 129,089 \pm \$ 4621$ from $1998 / 1999$ to $2008 / 2009$ (inflation-adjusted 2009 dollars). Estimated national total charges for all BAV and aortic surgery hospitalizations over this 12 -year period were $\$ 5.0$ billion, increasing from $\$ 119$ million in $1998 \quad(\sim \$ 156$ million inflation-adjusted 2009 dollars) to $\$ 1.18$ billion in 2009 .

\section{DISCUSSION}

These data demonstrate an increase in TAS in patients with BAV. There has been a steady increase in the proportion of patients with BAV undergoing AVR and concomitant surgery on the thoracic aorta. The proportion of patients with a diagnosis of TAA has paralleled the increase in TAS, suggesting a more liberal definition of aortic aneurysm associated with BAV. Greater use of tomographic radiology (eg, computed tomography or magnetic resonance imaging/angiography) and other imaging technologies may have contributed to this trend. A greater number of patients undergoing surgery for a primary indication of thoracic aortic disease with AVR performed for otherwise borderline aortic valve disease also may have a role. Given the smaller number of patients undergoing aortic surgery alone in the absence of dissection or coarctation, we suspect this represents a minority of the additional TAS. These findings do not simply reflect a universal trend of increasing use of cardiac procedures. For example, the population frequency of coronary bypass grafting surgery decreased between 2001/2002 and 2007/2008, whereas the use of percutaneous coronary intervention remained stable. ${ }^{34}$ The annual number of AVR operations performed in the United States increased modestly, only $28 \%$, between 1998 and 2005. ${ }^{29}$

Professional society guidelines, such as those from the American College of Cardiology (ACC) and American Heart Association (AHA), have progressively recommended a more liberal approach to thoracic aortic dilation in patients with BAV, similar to the approach suggested for patients with connective tissue disease. These recommendations are mainly based on expert consensus in the absence of more definitive outcomes data. The 1998 ACC/ AHA guidelines for the management of valvular heart disease suggested that aortic root replacement was generally indicated when the aortic diameter was $5 \mathrm{~cm}$ or more in the setting of aortic regurgitation compelling AVR. No specific note was made of any considerations for BAV disease. $^{21}$ The 2006 ACC/AHA guidelines for valvular heart disease specifically discuss BAV with dilated ascending aorta, noting a threshold for isolated intervention on an ascending aorta $5 \mathrm{~cm}$ or more in diameter, but lowering the bar for patients undergoing AVR for severe aortic stenosis or regurgitation to a threshold diameter of $4.5 \mathrm{~cm} .{ }^{35}$ The subsequent 2010 ACC/AHA guidelines on thoracic aortic disease recommend that elective aortic intervention is indicated for an aortic diameter of 4 to $5 \mathrm{~cm}$ for BAV (or any other genetically mediated aortic disorder), independent of aortic valve function. ${ }^{23}$ Some have suggested even lower thresholds. ${ }^{24}$ There is considerable controversy regarding these recommendations, and some investigators have pointed to a lack of direct evidence that BAV aortic disease is truly comparable to other genetic syndromes associated with thoracic aortic disease, such as Marfan syndrome. ${ }^{9,26,36}$ In any case, the timing of the shift in aortic surgery observed in the current report, occurring well before official changes in guideline documents, suggests 
TABLE 2. Descriptive statistics for patients with bicuspid aortic valve hospitalized in the United States for aortic valve or thoracic aortic surgery stratified by 2-year epoch between 1998 and 2009

\begin{tabular}{|c|c|c|c|c|c|c|c|c|c|c|c|c|c|}
\hline & \multicolumn{2}{|c|}{ 1998/1999 } & \multicolumn{2}{|c|}{$2000 / 2001$} & \multicolumn{2}{|c|}{$2002 / 2003$} & \multicolumn{2}{|c|}{$2004 / 2005$} & \multicolumn{2}{|c|}{$2006 / 2007$} & \multicolumn{2}{|c|}{ 2008/2009 } & \multirow[b]{2}{*}{$P$ valu } \\
\hline & $\% *$ & SE & $\%$ & SE & $\%$ & SE & $\%$ & SE & $\%$ & SE & $\%$ & SE & \\
\hline No. (n), weighted & 4556 & 571 & 5922 & 737 & 6464 & 635 & 8208 & 891 & 10,305 & 1095 & 14,960 & 2107 & $<.0001$ \\
\hline Age, y (mean, SEM) & 51.1 & 0.7 & 52.1 & 0.6 & 52.8 & 0.5 & 53.4 & 0.5 & 55.4 & 0.4 & 55.7 & 0.4 & $<.0001$ \\
\hline \multicolumn{14}{|l|}{ Age group, y } \\
\hline $18-30$ & 10.0 & 1.2 & 8.3 & 0.9 & 5.5 & 0.6 & 6.7 & 0.8 & 4.0 & 0.5 & 5.4 & 0.5 & \multirow[t]{5}{*}{$<.0001$} \\
\hline$>30-40$ & 12.7 & 1.4 & 11.4 & 1.1 & 16.0 & 1.2 & 9.5 & 0.8 & 10.2 & 0.8 & 8.3 & 0.5 & \\
\hline$>40-50$ & 23.2 & 1.4 & 22.2 & 1.2 & 20.3 & 1.2 & 20.7 & 1.0 & 17.8 & 1.0 & 15.4 & 0.8 & \\
\hline$>50-65$ & 32.6 & 1.5 & 36.0 & 1.5 & 34.1 & 1.3 & 41.1 & 1.1 & 41.2 & 1.1 & 42.4 & 0.9 & \\
\hline$>65$ & 21.5 & 1.6 & 22.1 & 1.6 & 24.1 & 1.2 & 21.9 & 1.3 & 26.8 & 1.1 & 28.5 & 1.2 & \\
\hline Gender ( $\%$ female $)$ & 26.3 & 1.6 & 27.5 & 1.2 & 29.6 & 1.3 & 26.1 & 1.1 & 26.5 & 1.0 & 26.6 & 0.8 & .42 \\
\hline Aortic dissection & 4.0 & 0.7 & 3.3 & 0.5 & 2.5 & 0.5 & 1.7 & 0.3 & 1.9 & 0.4 & 2.3 & 0.3 & .003 \\
\hline SBE & 4.0 & 0.6 & 4.5 & 0.7 & 3.8 & 0.5 & 3.7 & 0.5 & 3.2 & 0.4 & 2.9 & 0.4 & .009 \\
\hline Coarctation & 1.2 & 0.4 & 1.5 & 0.3 & 0.5 & 0.2 & 1.5 & 0.3 & 1.4 & 0.2 & 1.0 & 0.2 & .66 \\
\hline \multicolumn{14}{|l|}{ Comorbidities } \\
\hline 0 & 44.2 & 2.1 & 40.9 & 1.7 & 34.5 & 1.5 & 28.5 & 1.5 & 22.0 & 1.1 & 15.5 & 1.5 & \multirow[t]{4}{*}{$<.0001$} \\
\hline 1 & 36.1 & 1.8 & 34.8 & 1.3 & 34.6 & 1.2 & 38.5 & 1.2 & 33.1 & 1.1 & 30.4 & 1.3 & \\
\hline 2 & 13.2 & 1.1 & 17.7 & 1.2 & 20.3 & 1.0 & 20.1 & 1.0 & 26.0 & 1.0 & 26.6 & 1.1 & \\
\hline$\geq 3$ & 6.4 & 1.2 & 6.7 & 1.2 & 10.6 & 1.4 & 12.9 & 1.3 & 18.9 & 1.6 & 27.4 & 2.1 & \\
\hline Mechanical valve & 69.2 & 3.2 & 63.3 & 2.5 & 60.2 & 2.0 & 57.2 & 2.4 & 46.1 & 2.7 & 37.8 & 3.7 & $<.0001$ \\
\hline \multicolumn{14}{|l|}{ Type of surgery } \\
\hline AVR only & 85.0 & 1.6 & 83.2 & 1.7 & 79.4 & 1.8 & 73.6 & 2.0 & 71.8 & 1.7 & 69.0 & 1.5 & \multirow[t]{3}{*}{$<.0001$} \\
\hline AVR with TAS & 12.4 & 1.4 & 13.9 & 1.6 & 17.3 & 1.4 & 22.8 & 1.8 & 24.6 & 1.6 & 27.5 & 1.5 & \\
\hline Isolated TAS & 2.5 & 0.6 & 2.8 & 0.5 & 3.3 & 0.6 & 3.6 & 0.5 & 3.5 & 0.5 & 3.5 & 0.4 & \\
\hline CABG & 22.3 & 1.8 & 21.5 & 1.3 & 21.3 & 1.3 & 19.3 & 1.3 & 22.1 & 1.3 & 22.8 & 0.8 & .43 \\
\hline Mitral valve repair & 0.5 & 0.3 & 1.3 & 0.3 & 1.0 & 0.3 & 2.4 & 0.4 & 1.8 & 0.3 & 2.0 & 0.4 & .004 \\
\hline TAA & 15.0 & 1.8 & 16.3 & 1.6 & 22.2 & 1.6 & 27.0 & 1.7 & 28.6 & 1.5 & 33.4 & 1.6 & $<.0001$ \\
\hline Teaching hospital & 80.7 & 4.0 & 78.0 & 3.8 & 71.8 & 4.1 & 74.9 & 3.8 & 69.1 & 4.2 & 75.5 & 4.1 & .33 \\
\hline \multicolumn{14}{|l|}{ Hospital region } \\
\hline Northeast & 21.8 & 4.9 & 19.8 & 4.4 & 14.1 & 2.9 & 20.6 & 5.6 & 20.2 & 5.2 & 18.4 & 4.1 & \multirow[t]{4}{*}{.30} \\
\hline Midwest & 29.7 & 5.7 & 24.5 & 4.8 & 25.6 & 4.1 & 30.6 & 4.9 & 26.5 & 4.4 & 39.9 & 8.0 & \\
\hline South & 28.2 & 6.1 & 37.1 & 6.7 & 32.3 & 4.8 & 26.8 & 4.2 & 31.7 & 4.8 & 23.1 & 4.3 & \\
\hline West & 20.3 & 4.6 & 18.6 & 4.0 & 28.0 & 4.4 & 22.0 & 4.0 & 21.5 & 4.0 & 18.6 & 3.5 & \\
\hline Private insurance & 67.6 & 2.4 & 68.1 & 1.6 & 64.9 & 1.6 & 66.6 & 1.5 & 62.9 & 1.4 & 60.1 & 1.2 & $<.0001$ \\
\hline Died & 2.5 & 0.6 & 2.0 & 0.4 & 1.7 & 0.4 & 1.3 & 0.3 & 1.3 & 0.3 & 1.5 & 0.2 & .02 \\
\hline
\end{tabular}

$S E$, Standard error; $S E M$, standard error of the mean; $S B E$, subacute bacterial endocarditis; $A V R$, aortic valve replacement or repair; $T A S$, thoracic aortic surgery; $C A B G$, coronary artery bypass grafting; TAA, thoracic aortic aneurysm. *Data are presented as \% with standard error \% unless otherwise specified.

the guidelines may have trailed rather than led clinical practice in this instance.

We also observed an increase in the number of isolated AVR for patients with a diagnosis of BAV. We think it unlikely that this represents a true increase in aortic valve disease in patients with BAV, but rather a combination of factors including improved diagnosis of BAV related to imaging technology and more widespread awareness that BAV is a common cause of aortic valve stenosis and regurgitation in adults.

Improved surgical techniques and reduced morbidity and mortality over the last 2 decades may have decreased patient or provider reluctance to proceed with surgery. ${ }^{37-39}$ It is notable that in-hospital mortality for patients undergoing AVR alone was similar to AVR and concomitant TAS in our population. This occurred despite the longer myocardial ischemic and cardiopulmonary bypass time (and sometimes circulatory arrest) associated with the addition of aortic surgery. ${ }^{40,41}$ Although our observations may reflect unmeasured differences in patient characteristics, adjustments for available potential cofounders did not alter this finding. Although counterintuitive, our results mirror a prior single-center report that applied extensive propensity score adjustments using more detailed clinical data and also found no difference for in-hospital mortality. ${ }^{41}$ Our data cannot provide insight on more subtle functional effects or postdischarge outcomes, which also are clearly important for consideration of the risks and benefits of aortic intervention.

The present data do not support a value judgment on the trends described. Rather, the results highlight the importance of obtaining definitive data to guide clinicians in the complex risk/benefit considerations involved. First, the number of patients affected by these decisions is increasing 


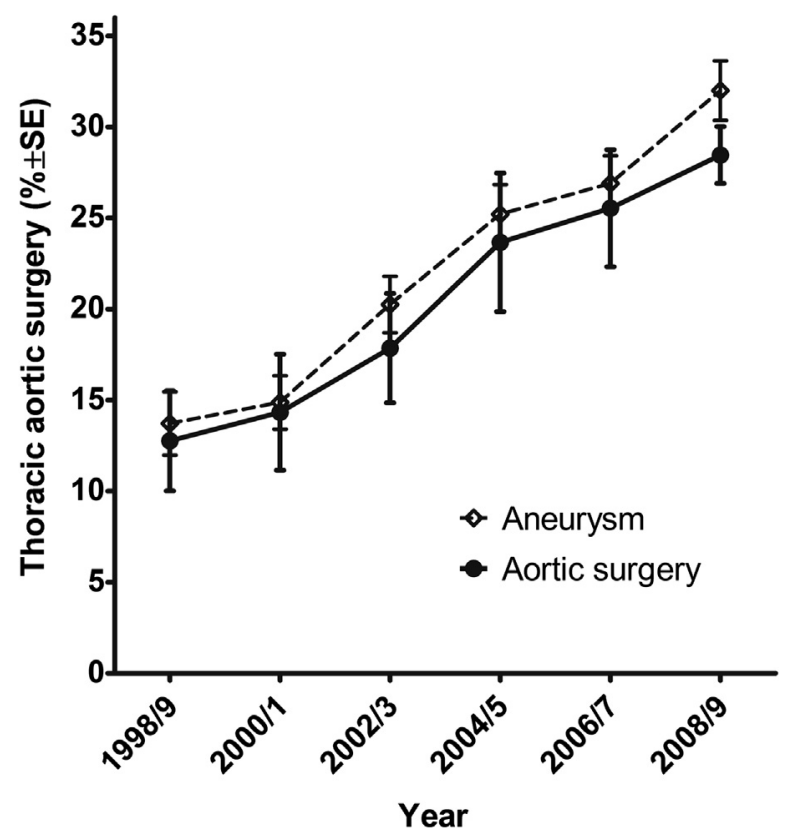

FIGURE 2. Temporal trend in the proportion of hospitalizations in which patients with BAV receiving AVR underwent concomitant TAS $(\% \pm$ standard error \%), 1998-2009. The increase in aortic surgery was closely paralleled by an increase in diagnosis of TAA (dotted line). $S E$, Standard error.

dramatically. Second, clinicians seem to be making major changes in their approach to these issues based on indirect evidence along with shifting expert consensus. Finally,

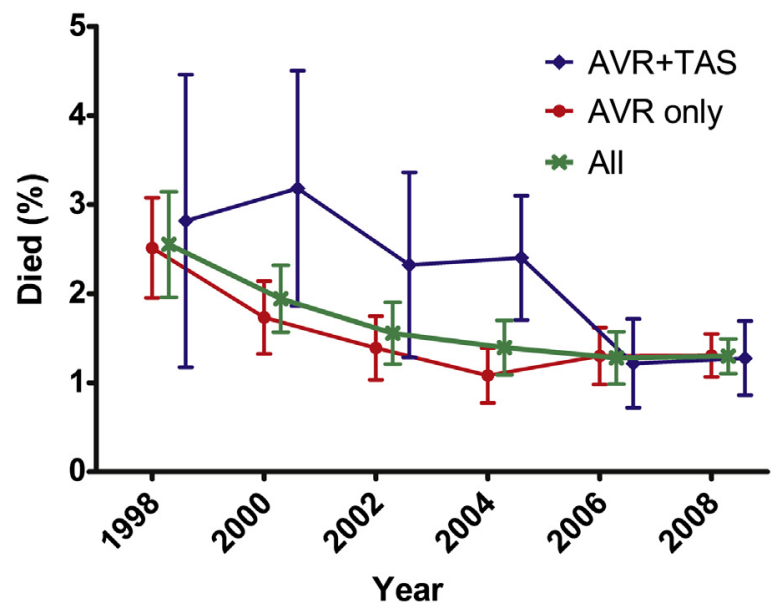

FIGURE 4. In-hospital mortality, by 2-year time period, for patients undergoing isolated AVR and patients undergoing concomitant TAS. There was a trend toward lower mortality over time for all groups. Data for isolated TAS are not shown because of the smaller number of cases/deaths, as well as distinct patient characteristics (ie, higher dissection and coarctation frequency). Error bars represent standard error \%. AVR, Aortic valve replacement or repair; TAS, thoracic aortic surgery.

these interventions constitute an increasing financial burden. Taken together, these findings make a strong argument that the growing population at risk and important ramifications of the clinical question merit collection of more rigorous outcomes data on potential approaches toward TAS in these patients.
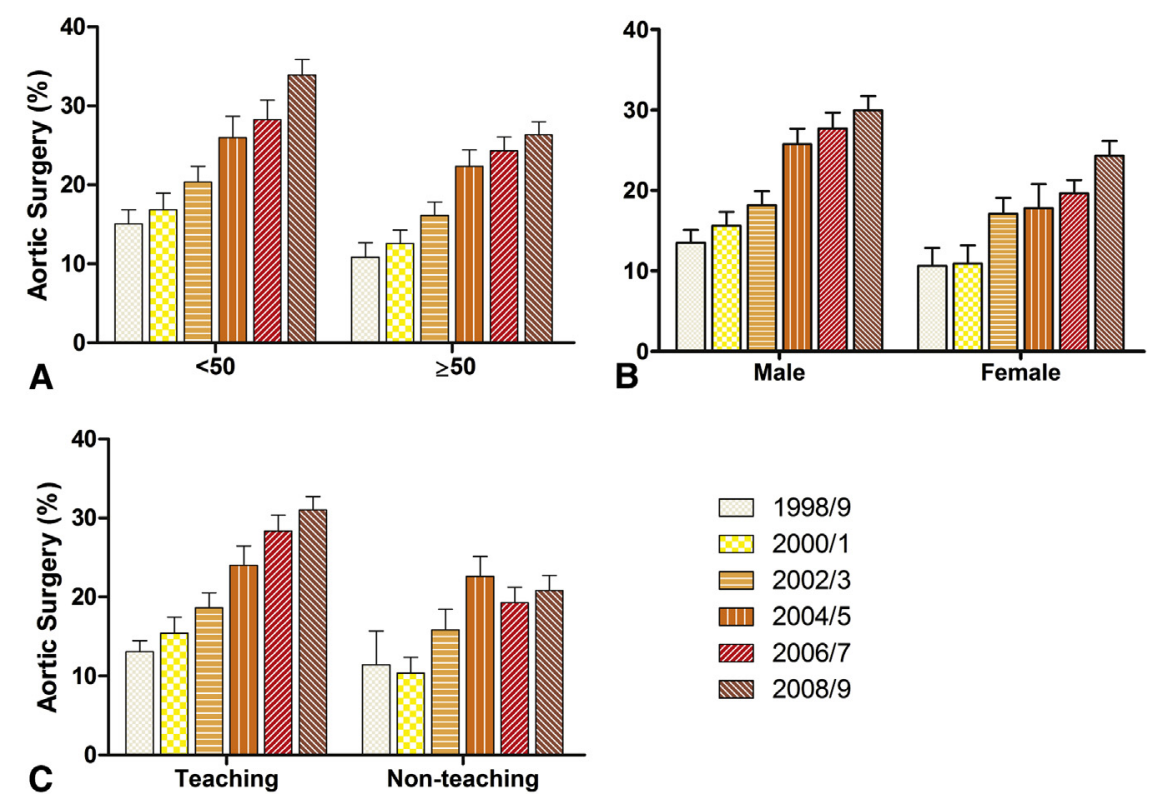

FIGURE 3. Subgroup analysis of the proportion of patients undergoing AVR that included concomitant TAS for each time epoch. A, Patients aged less than 50 years or 50 or more years. A greater proportion of younger patients underwent aortic surgery, at an increasing frequency of the study duration. B, Gender. Men more frequently underwent aortic surgery, at an increasing frequency of the study duration. C, Type of hospital. Concomitant aortic surgery was performed more frequently at teaching hospitals, at an increasing frequency of the study duration. Nonteaching hospitals did not show a similar trend to perform concomitant aortic surgery over the study duration. There seemed to be a more consistent and prominent trend to a greater proportion of aortic surgeries in teaching hospitals. 


\section{Study Limitations}

Our findings must be interpreted in light of methodological constraints. Large administrative databases have advantages in terms of sample size and inclusiveness, but also pose limitations on the analysis. International Classification of Diseases 9th Revision codes provide limited clinical detail, and the validity is unknown for most diagnoses studied. There were no data on aortic size or other detailed clinical data beyond diagnostic and procedural codes. However, the population studied and clinical management are similar to those reported in the literature for patients with BAV undergoing AVR. ${ }^{41}$ We did not account for changes in the size of the general population, but these changes are smaller than those described $(\sim 11 \%$ increase in the US adult population from 2000 to 2009). In any case, such shifts would only affect the absolute number of procedures and not the relative proportions of patients managed with a given surgical strategy. There is no mechanism linking multiple admissions for a specific patient, although this should not pose a challenge to the validity of the main finding of the study: a shift in approach to the thoracic aorta in this population. Although general trends and correlates of such changes in management can be described, the available data do not permit extensive analysis of the underlying causes. Data on hospital charges reflect only inpatient charges and cannot be directly translated to actual costs and exclude any associated outpatient costs related to the diagnoses and procedures studied.

\section{CONCLUSIONS}

The annual number of AVR and TAS performed in patients with BAV increased between 1998 and 2009. Although the number of isolated AVRs has grown, the most dramatic shift has been in the application of TAS. These findings likely reflect a greater sensitivity to aortic disease in this population. Optimal management of bicuspid aortopathy remains undefined, however, and there is a pressing need for rigorous investigation on the clinical approach to the aorta in patients with BAV.

The authors thank Lilamarie Moko for help in preparing and reviewing the manuscript, and Gabriele Egidy Assenza for insightful review of the manuscript.

\section{References}

1. Roberts WC. The congenitally bicuspid aortic valve. A study of 85 autopsy cases. Am J Cardiol. 1970;26:72-83.

2. Larson EW, Edwards WD. Risk factors for aortic dissection: a necropsy study of 161 cases. Am J Cardiol. 1984;53:849-55.

3. Braverman AC, Guven H, Beardslee MA, Makan M, Kates AM, Moon MR. The bicuspid aortic valve. Curr Probl Cardiol. 2005;30:470-522.

4. Keane MG, Wiegers SE, Plappert T, Pochettino A, Bavaria JE, Sutton MG. Bicuspid aortic valves are associated with aortic dilatation out of proportion to coexistent valvular lesions. Circulation. 2000;102(19 Suppl 3):III35-9.

5. Yasuda H, Nakatani S, Stugaard M, et al. Failure to prevent progressive dilation of ascending aorta by aortic valve replacement in patients with bicuspid aortic valve: comparison with tricuspid aortic valve. Circulation. 2003;108(Suppl 1): II291-4.
6. Biner S, Rafique AM, Ray I, Cuk O, Siegel RJ, Tolstrup K. Aortopathy is prevalent in relatives of bicuspid aortic valve patients. J Am Coll Cardiol. 2009;53: 2288-95.

7. Tadros TM, Klein MD, Shapira OM. Ascending aortic dilatation associated with bicuspid aortic valve: pathophysiology, molecular biology, and clinical implications. Circulation. 2009;119:880-90.

8. Michelena HI, Khanna AD, Mahoney D, et al. Incidence of aortic complications in patients with bicuspid aortic valves. JAMA. 2011;306:1104-12.

9. Sundt TM 3rd. Replacement of the ascending aorta in bicuspid aortic valve disease: where do we draw the line? J Thorac Cardiovasc Surg. 2010;140(6 Suppl): S41-4; discussion S5-51.

10. Ransohoff J. VI. The extirpation of aneurisms. Ann Surg. 1894;19:78-84.

11. Bergqvist D. Historical aspects on aneurysmal disease. Scand J Surg. 2008;97: 90-9.

12. Braverman AC. Aortic involvement in patients with a bicuspid aortic valve Heart. 2011;97:506-13.

13. Borger MA, Preston M, Ivanov J, et al. Should the ascending aorta be replaced more frequently in patients with bicuspid aortic valve disease? J Thorac Cardiovasc Surg. 2004;128:677-83.

14. Wald O, Korach A, Shapira OM. Should aortas in patients with bicuspid aortic valve really be resected at an earlier stage than tricuspid? PRO. Cardiol Clin. 2010;28:289-98

15. Leone O, Biagini E, Pacini D, et al. The elusive link between aortic wall histology and echocardiographic anatomy in bicuspid aortic valve: implications for prophylactic surgery. Eur J Cardiothorac Surg. 2012;41:322-7.

16. Elefteriades JA. Indications for aortic replacement. J Thorac Cardiovasc Surg 2010;140(6 Suppl):S5-9; discussion S45-51.

17. Pisano C, Maresi E, Balistreri CR, et al. Histological and genetic studies in patients with bicuspid aortic valve and ascending aorta complications. Interact Cardiovasc Thorac Surg. 2012;14:300-6.

18. Prapa M, Ho SY. Risk stratification in bicuspid aortic valve disease: still more work to do. Eur J Cardiothorac Surg. 2012;41:327-8.

19. Coady MA, Rizzo JA, Hammond GL, et al. What is the appropriate size criterion for resection of thoracic aortic aneurysms? J Thorac Cardiovasc Surg. 1997;113: 476-91; discussion 89-91.

20. Kouchoukos NT, Dougenis D. Surgery of the thoracic aorta. N Engl J Med. 1997 336:1876-88.

21. Bonow RO, Carabello B, de Leon AC, et al. ACC/AHA Guidelines for the Management of Patients With Valvular Heart Disease. Executive Summary. A report of the American College of Cardiology/American Heart Association Task Force on Practice Guidelines (Committee on Management of Patients With Valvular Heart Disease). J Heart Valve Dis. 1998;7:672-707.

22. Elefteriades JA. Natural history of thoracic aortic aneurysms: indications for surgery, and surgical versus nonsurgical risks. Ann Thorac Surg. 2002;74:S1877-80 discussion S92-8.

23. Hiratzka LF, Bakris GL, Beckman JA, et al. 2010 ACCF/AHA/AATS/ACR/ ASA/SCA/SCAI/SIR/STS/SVM guidelines for the diagnosis and management of patients with Thoracic Aortic Disease: a report of the American College of Cardiology Foundation/American Heart Association Task Force on Practice Guidelines, American Association for Thoracic Surgery, American College of Radiology, American Stroke Association, Society of Cardiovascular Anesthesiologists, Society for Cardiovascular Angiography and Interventions, Society of Interventional Radiology, Society of Thoracic Surgeons, and Society for Vascular Medicine. Circulation. 2010;121:e266-369.

24. Isselbacher EM. Thoracic and abdominal aortic aneurysms. Circulation. 2005 111:816-28

25. Elefteriades JA. Editorial comment: should aortas in patients with bicuspid aortic valve really be resected at an earlier stage than those in patients with tricuspid valve? Cardiol Clin. 2010;28:315-6.

26. Guntheroth WG. A critical review of the American College of Cardiology/American Heart Association practice guidelines on bicuspid aortic valve with dilated ascending aorta. Am J Cardiol. 2008;102:107-10.

27. Opotowsky AR, Siddiqi OK, Webb GD. Trends in hospitalizations for adults with congenital heart disease in the U.S. J Am Coll Cardiol. 2009; 54:460-7.

28. Schermerhorn ML, Giles KA, Hamdan AD, Dalhberg SE, Hagberg R, Pomposelli F. Population-based outcomes of open descending thoracic aortic aneurysm repair. J Vasc Surg. 2008;48:821-7.

29. Barnett SD, Ad N. Surgery for aortic and mitral valve disease in the United States: a trend of change in surgical practice between 1998 and 2005. J Thorac Cardiovasc Surg. 2009;137:1422-9. 
30. Opotowsky AR, Siddiqi OK, D'Souza B, Webb GD, Fernandes SM, Landzberg MJ. Maternal cardiovascular events during childbirth among women with congenital heart disease. Heart. 2012;98:145-51.

31. Elixhauser A, Steiner C, Harris DR, Coffey RM. Comorbidity measures for use with administrative data. Med Care. 1998;36:8-27.

32. Healthcare Cost and Utilization Project Nationwide Inpatient Sample. 20072009. Available at: www.hcup-us.ahrq.gov/nisoverview.jsp. Accessed March 21, 2012.

33. Nationwide Inpatient Sample Database Documentation. Available at: http:// www.hcup-us.ahrq.gov/db/nation/nis/nisdbdocumentation.jsp. Accessed March 21, 2012.

34. Epstein AJ, Polsky D, Yang F, Yang L, Groenveld PW. Coronary revascularization trends in the United States, 2001-2008. JAMA. 2011;305:1769-76.

35. Bonow RO, Carabello BA, Kanu C, et al. ACC/AHA 2006 guidelines for the management of patients with valvular heart disease: a report of the American College of Cardiology/American Heart Association Task Force on Practice Guidelines (writing committee to revise the 1998 Guidelines for the Management of Patients With Valvular Heart Disease): developed in collaboration with the Society of Cardiovascular Anesthesiologists: endorsed by the Society for Cardio- vascular Angiography and Interventions and the Society of Thoracic Surgeons Circulation. 2006;114:e84-231.

36. Coady MA, Stockwell PH, Robich MP, Poppas A, Sellke FW. Should aortas in patients with bicuspid aortic valve really be resected at an earlier stage than tricuspid? CON. Cardiol Clin. 2010;28:299-314.

37. Olsson C, Thelin S, Stahle E, Ekbom A, Granath F. Thoracic aortic aneurysm and dissection: increasing prevalence and improved outcomes reported in a nationwide population-based study of more than 14,000 cases from 1987 to 2002 . Circulation. 2006;114:2611-8.

38. Chiesa R, Melissano G, Civilini E, de Moura ML, Carozzo A, Zangrillo A. Ten years experience of thoracic and thoracoabdominal aortic aneurysm surgical repair: lessons learned. Ann Vasc Surg. 2004;18:514-20.

39. Booher AM, Eagle KA. Diagnosis and management issues in thoracic aortic aneurysm. Am Heart J. 2011;162:38-46.

40. Nazer RI, Elhenawy AM, Fazel SS, Garrido-Olivares LE, Armstrong S, David TE. The influence of operative techniques on the outcomes of bicuspid aortic valve disease and aortic dilatation. Ann Thorac Surg. 2010;89:1918-24.

41. Svensson LG, Kim KH, Blackstone EH, et al. Bicuspid aortic valve surgery with proactive ascending aorta repair. J Thorac Cardiovasc Surg. 2011;142:622-9, 9.e1-3. 